\title{
Una sociedad basada en la carrera de armamentos
}

\author{
Por Joan TRAVÉ
}

\section{INTRODUCCION}

En un curso $(*)$ sobre las grandes injusticias de hoy se ha analizado el tema del empobrecimiento progresivo del Tercer Mundo y el tema del paro. La pobreza o la miseria de casi dos tercios de la Humanidad. Y así, en nuestra época, cuando parece que se ha realizado la utopía de un mundo que ha alcanzado un nivel de desarrollo tecnológico suficiente para satisfacer las necesidades básicas de la Humanidad, el reparto desigual hace, no obstante, que sólo un tercio de esta humanidad lo pueda disfrutar. El $70 \%$ de la población humana está condenada a limitarse a sobrevivir o a morirse de hambre.

Al mismo tiempo, gran parte de los esfuerzos que tendrían que dedicarse a la solución de este problema van orientados hacia una creciente y dramática carrera de armamentos que, si nos puede llevar al exterminio de la raza humana, al mismo tiempo imposibilita la urgencia absoluta de eliminar el subdesarrollo. $Y$ así, mientras $\mathbf{4 0}$ millones de personas se mueren cada año por falta de nutrición, se dedica un millón y medio de dólares por minuto a la producción de armamentos. Y mientras los Estados han adquirido conciencia del peligro que representa por ejemplo la droga o el terrorismo, estos mismos Estados se vanaglorian de su capacidad para fabricar y exportar las armas más avanzadas.

El momento actual de nuestro país es una gran responsabilidad histórica. Nuestra integración definitiva a la OTAN supone un reforzamiento de la política de bloques, del enfrentamiento y de la creciente e imparable carrera armamentista. Una política distanciada de los bloques y que no quiere como enemigos ni a la Unión Soviética ni a los Estados Unidos, colaboraría a aumentar la paz y la distensión.

Se impone encontrar de nuevo la sensatez, la razón y el sentido de una sociedad que se ha convertido en irracional dentro de su pretendida

$\left({ }^{*}\right)$ Este artículo forma parte del curso que CRISTIANISME ! JUSTITICA Impartió en BarceIona en $1984-85$ y publicó en los cuadernos 9 y 10 bajo el título GRANS INJUSTICIES D'AVUI. 
racionalidad. De una sociedad que dice querer salvar la paz y eliminar la miseria y dedica sus esfuerzos al crecimiento y perfeccionamiento de unas armas que, no nos engañemos, serán utilizadas alguna vez, como siempre ha pasado en el curso de la Historia.

Se impone una información y un debate para movilizar la sociedad y presionar a los poderes públicos nacionales e internacionales con toda la fuerza y la imaginación posibles. Hay que luchar contra los argumentos capciosos que nos dicen que de todas maneras siempre existirá otro país que venderá las armas, que la no fabricación de armamento aumentará el paro y que sólo se venden armas para la defensa. Detrás de todo esto se esconden intereses económicos y políticos que se nutren del negocio de las armas y que difícilmente buscarán alternativas para hacer una readaptación de las industrias bélicas con fines pacíficos.

Tenemos que relativizar al máximo la hipotética defensa y el peligro de hegemonía de un bloque cuando la supervivencia y los mínimos para una vida humana están en peligro.

Quizá nunca una responsabilidad tan clara y tan pesada ha caído sobre las espaldas de una generación, porque las alternativas están bien definidas: optamos por alimentar un futuro de muerte u optamos por alimentar un futuro de vida.

Estas líneas quieren recoger algunos elementos significativos que ayuden a esta reflexión. Lo haremos bajo los siguientes apartados:

1. La irracionalidad de la situación actual.

II. La carrera armamentista: peligro de exterminio.

III. La carrera armamentista: una muerte lenta para el Tercer Mundo.

IV. La carrera armamentista, empobrecimiento para los países desarrollados: el paro y la inflación.

V. Caminos de ruptura y posibles alternativas.

VI. Conclusión.

\section{LA IRRACIONALIDAD DE LA SITUACION ACTUAL}

Para explicar qué queremos decir por una sociedad irracional en su totalidad aprovecharé la parábola que Roger Garaudy (1) nos explica bajo el título de "La parábola del molino del diablo", para hacernos ver en lenguaje de parábola a dónde puede llevar una sociedad basada en el mito del crecimiento y mucho más cuando este crecimiento está fundamentado en el crecimiento armamentístico.

En un país"altamente desarrollado", nos dice, el gobierno ha reconocido el derecho a todo individuo, derecho muy de àcuerdo con la libertad, de ir armado.

(1) Roger Garandy: La alternativa, Madrid, "Cuadernos para el Diálogo", 1977. Págs. 84 y ss. 
El uso de las armas personales lleva a una prosperidad de la industria armamentista sin precedentes. El mercado parece no tener límites. Surgen modelos de todo tipo: de bolsillo, de máxima precisión, etc. Crece el nivel de vida, aumentan las ventas al exterior. Se produce un "boom" de las industrias accesorias y de toda la siderurgia.

El "boom" combinado con el nerviosismo, la inseguridad y la tensión existentes fomentan la expansión de otros setores: cirugía, psiquiatría... Incluso las Ciencias Sociales. Se abren nuevas posibilidades de investigación, se hacen estadísticas de una precisión admirable, hasta se llega a descubrir la "ley logarítmica del exterminio" que permite prever cuándo el último hombre apuntando a su vecino disparará el último tiro de muerte.

El gobierno está contento con la realización de los sueños de la economía del crecimiento: moneda sólida, aumento de la venta, nuevos mercados.

En clave de parábola, este ejemplo que nos propone R. Garaudy podríamos decir que es el paradigma de una sociedad que "funciona", de una sociedad racionalizada, pero que es irracional en su totalidad. Se preocupa del funcionamiento, pero no se preocupa del sentido ni adónde va a parar esta sociedad. Y éste es el problema actual del mundo: una carrera de armamentos que arruina a las naciones y que parece que no se podrán utilizar si no se quiere provocar el exterminio de la humanidad. Este parece ser el "sentido" de nuestra sociedad.

\section{LA CARRERA ARMAMENTISTA: PELIGRO DE EXTERMINIO}

\section{Efectos de una guerra nuclear}

La novedad de una guerra nuclear es la ilimitación de los efectos en el espacio y en el tiempo. Es una guerra que puede exterminar a la humanidad (genocidio), a la ecología (ecocidio) y al cosmos (cosmocidio). Ya no sería una guerra contra una clase como otras veces se ha dicho, sino contra la misma especie. Se puede hacer una gradación de efectos, como F. Urbina (2), de la siguiente manera:

a) Efectos inmediatos. El momento de la explosión y las horas siguientes en un radio de acción de pocos $\mathrm{Km}$. Por ejemplo en Hiroshima murieron 85.000 personas en el momento de la explosión y 70.000 , las 48 horas posteriores.

b) Efectos intermedios. Efectos que se producen días o semanas después y hasta distancias de unos centenares de $\mathrm{Km}$.

c) Efectos a largo plazo. Efectos que se producen en una distancia y en un tiempo indenifidos. Los límites pueden ser desde la destrucción del planeta entero hasta la desintegración genética.

(2) Fernando Urbina: La guerra nuclear. Madrid. Editorial Popular, 1984. Págs. 16 y ss. 
Hoy en día se habla frecuentemente del "invierno nuclear" un enfriamiento general que podría provocar una nueva glaciación.

Con lo que hemos dicho ya se ve que una guerra como ésta tiene pocos puntos de contacto con las guerras que a lo largo de la historia se ha intentado justificar con teorías como las de la "guerra justa". En aquellas guerras siempre existía un elemento clave para justificarlas: el "control de los daños". Ahora es un acto absolutamente incontrolado. Puesta en marcha la dinámica, los resultados escapan a toda previsibilidad, dejan de ser humanos.

2. Justificaciones de la carrera armamentista: la disuasión, el equilibrio y la inestabilidad estratégica

La disuasión quiere significar sencillamente espantar al otro para que no ataque. Es la estrategia del terror. Ha tenido una formulación famosa con el MAD (Mutua destrucción asegurada). Significa una capacidad de respuesta suficientemente fuerte después de recibir el primer golpe. Más adelante se habló del OVERKILL (exceso de capacidad mortífera). Quiere significar tener muchas más armas que las necesarias para una respuesta adecuada, para estar siempre seguros de la mutua destrucción asegurada, es decir, que el mejoramiento cualitativo y cuantitativo del adversario nos mantiene siempre el MAD. Esta es fundamentalmente la teoría de la disuasión que mantienen las dos superpotencias y que extienden como un paraguas a sus aliados.

El equilibrio o la paridad de armamentos se ha demostrado que es una cosa irrealizable. Se ha convertido en una carrera de las grandes potencias para no quedarse atrás.

Un problema que nunca se ha solucionado: cómo controlar el armamento de las superpotencias. Y una segunda cuestión fundamental: ¿quién tiene la superioridad? Con todas las dificultades que las comparaciones comportan, R. Grasa (3) puede afirmar "que nadie de buena fe puede negar que la práctica totalidad de expertos occidentales admite la existencia de una disimetría a escala mundial desfavorable a la URSS". Aunque según el autor la URSS tendría superioridad en lanzadores y potencia explosiva, la superioridad USA es clara en cabezas nucleares, precisión y vulnerabilidad, ya que los Estados Unidos tienen, a diferencia de los rusos, instalada mayoritariamente su potencia nuclear en los submarinos y bombarderos, mucho menos vulnerables que las instalaciones rusas, predominantemente instaladas en la superficie.

De todas formas, a pesar de las cifras que se pueden mezclar, parece ser que hay un cierto consenso entre los expertos militares en que el número de misiles balísticos intercontinentales, bombarderos nucleares y misiles instalados en los submarinos pueden producir un nivel de daños en una primera réplica que es inaceptable por una u otra superpotencia.

(3) Rafael Grasa: Las superpotencias y su política de rehenes. "El País", 6 de diciembre de 1984. 
La pregunta constante de la carrera nuclear es qué puede pasar cuando la política de la disuasión y de equilibrio se rompan.

Hoy en día, nos dice C. Alonso Zaldívar (4), el incremento de los programas de modernización de sistemas de armamentos tiende a producir: a) un incremento del "overkill"; b) disminución del nivel de mutua destrucción asegurada. Esta paradoja tiene una explicación. Cada día aumenta la precisión de las armas nucleares, de tal manera que, al final de la década, las instalaciones de misiles tendrán un índice de vulnerabilidad del 100 por 100, a pesar de que siempre quede la respuesta por los submarinos.

Pero la vulnerabilidad de los misiles balísticos intercontinentales (ICBMS) situará a los arsenales de las dos potencias dentro de una relación de capacidad destructiva-vulnerabilidad que dará a quien dispare primero una ventaja enorme. Esto es la "inestabilidad estratégica"? Con esta situación es probable que se presente más de una vez la necesidad de decidir urgentemente si se va o no a la guerra.

El mismo Zaldívar explica un escenario posible de guerra nuclear presentado por el grupo de estudios nucleares de Harvard, que, como dice el autor antes citado, no son precisamente pacifistas.

Según este "escenario", los satélites de la inteligencia americana detectan un barco ruso desembarcando misiles crucero en Cuba. El Jefe de Estado soviético dice que los retirará si la NATO retira los que ha desplegado en Europa.

El presidente americano decide atacar al barco por la noche. "No teníamos otra solución", dirá. Por el contrario, teníamos la amenaza en las fronteras. De hecho era un ataque limitado al barco para demostrar su posición de fuerza.

La información que recibe Moscú es que los americanos están a punto de comenzar un ataque nuclear. Hacen los cálculos y llegan a la conclusión que si USA ataca primero destruirá sus ICBM y producirá unos 100 millones de muertos. Si atacan los rusos primero, la represalia sólo producirá unos 10 o 20 millones de muertos o, incluso, falta de represalia.

De hecho la URSS se equivocaba. La guerra no era inevitable. Pero por miedo disparan primero. Morirán 35 millones de americanos inmediatamente y la respuesta matará 25 millones de rusos.

Los autores dicen que este escenario es perfectamente "plausible", y que una futura guerra nuclear por "malentendidos" será más probable que una guerra nuclear claramente premeditada.

\section{Puntos significativos de la carrera armamentista}

Si ponemos como punto de partida la bomba de Hiroshima, lo que ha seguido después no ha sido más que el desarrollo de aquella primera irra-

(4) Carlos Alonso Zaldivar: Guerra y Paz en el mundo nuclear. "Misión Abierta", septiem. bre 1983. Págs. 35 y 36. 
cionalidad. Hemos tenido un crecimiento en espiral del armamento, tanto desde un punto de vista cualitativo o de perfección tecnológica, como desde un punto de vista cuantitativo o de capacidad explosiva acumulada (5).

De los años cuarenta a los sesenta pasamos de los bombarderos $\mathrm{B}$ 29 (Hiroshima) a los B-52, con capacidad intercontinental.

En la década de los sesenta aparecen los grandes misiles intercontinentales que puede llevar una cabeza nuclear a $12.000 \mathrm{Km}$.

En los inicios de los setenta aparecen los M.I.R.V. o misiles con muchas cabezas, cada uno programable por objetivos diferentes.

En los ochenta, los presupuestos de más de 300.000 millones de dólares que lanza Ronald Reagan suponen progresos espectaculares, como el proyecto $\mathrm{MX}$, donde dentro de unos subterráneos enormes correrian centenares de trenes portadores de misiles que pueden disparar desde las infinitas aberturas y que tienen una probabilidad mínima de ser destruidos, hasta llegar a la llamada guerra de las galaxias.

Si lo miramos desde un punto de vista cuantitativo se nos dice que entre las dos superpotencias acumulan veinte mil megatones. Si, según los estrategas norteamericanos, con 400 megatones habría suficiente para provocar 30 millones de muertos en la URSS y dejar el país irreversiblemente destruido, parece absolutamente irracional la acumulación de 10.000 megatones.

\section{LA CARRERA ARMAMENTISTA: UNA MUERTE LENTA PARA EL TERCER MUNDO}

El crecimiento constante de los gastos militares y de los stocks de armamentos es un tema que se ha repetido tantas veces que acaba por ser un dato más de la vida cotidiana.

Cifras como 700 a 750.000 millones de dólares dedicadas en un año a la industria armamentística, casi 1 millón y medio por minuto, ya forman parte de nuestra cultura.

El arsenal mundial escapa a la imaginación: 16.000 millones de toneladas de TNT. En la Segunda Guerra Mundial con 3 millones de toneladas se produjeron 50 millones de muertos. Cada ciudadano USA dedica 130.000 ptas. anuales al presupuesto militar. El precio de un submarino nuclear cubriría el presupuesto de educación de 28 países en vías de desarrollo o la educación básica de 160 millones de niños (6).

Según la FAO, las tendencias actuales hasta el año 2000 incrementarán gravemente la población desnutrida hasta 600-650 millones. Y cada día mueren 40.000 niños de hambre en el mundo. El Consejo Mundial de

(5) Cfr. de manera más desarrollada en F. Urbina, o. c. págs. 46 y ss.

(6) Datos de la Fundación Rockefeller recogidas en Mariano Aguirre: De Hiroshima a los euromisiles. Madrid, Tecnos, 1984. Págs, 155, ss. 
la Alimentación indica que haría falta invertir 4.000 millones de dólares anuales - menos de la octava parte del presupuesto de investigación y desarrollo militar de un año- a fin de que dieran, durante los próximos quince años, ingresos activos productivos, para que cerca de 500 millones de personas puedan satisfacer las necesidades mínimas hasta finales de siglo.

Este tema de la conexión armamentismo-desarrollo es un tema fundamental si no pensamos únicamente el problema desde nuestra optica, desde "nuestra" paz, desde la paz de los acomodados que sería un lujo, un privilegio, conseguido a espaldas de la mayoría de la humanidad.

Es imposible elaborar una reforma de las leyes internacionales de intercambio, es imposible hablar de un NOEl, si la industria bélica sigue en el centro de las voluntades políticas que dominan el mundo. El desarrollo de los países más pobres no se puede hacer in las transferencias de recursos del Norte, y esto no es posible debido a los gastos militares.

Al mismo tiempo los países subdesarrollados aumentan exageradamente los gastos militares, como se puede ver en el gráfico 1.

GASTOS MILITARES DE LOS PAISES SUBDESARROLLADOS (en millones de dólares a precios constantes de 1979)

\begin{tabular}{lcc}
\hline & Gastos & $\begin{array}{c}\text { Por ciento de los gastos } \\
\text { de todo el mundo }\end{array}$ \\
1972 & 32.980 & 7,9 \\
1973 & 37.296 & 8,8 \\
1974 & 48.074 & 11,0 \\
1975 & 56.034 & 12,4 \\
1976 & 63.946 & 14,0 \\
1977 & 63.630 & 13,7 \\
1978 & 66.085 & 13,8 \\
1979 & 67.838 & 13,7 \\
1980 & 71.316 & 14,1 \\
1981 & 81.281 & 15,6 \\
\hline
\end{tabular}

Fuente: SIPRI; Yearbook, 1982, p. 140.

\section{Gráfico 1}

Las armas se compran cada vez más caras comparativamente con los precios de venta de las primeras materias, porque el deterioramiento de los términos de cambio y el intercambio desigual funcionan plenamente en el negocio de las armas.

La adquisición de armas representa para muchos países la tecnificación de la represión y la reproducción del ciclo pobreza-represiónmilitarización, como se ven en el gráfico 2 (7).

(7) Cit. en M. Aguirre, o, c. pág. 160. 


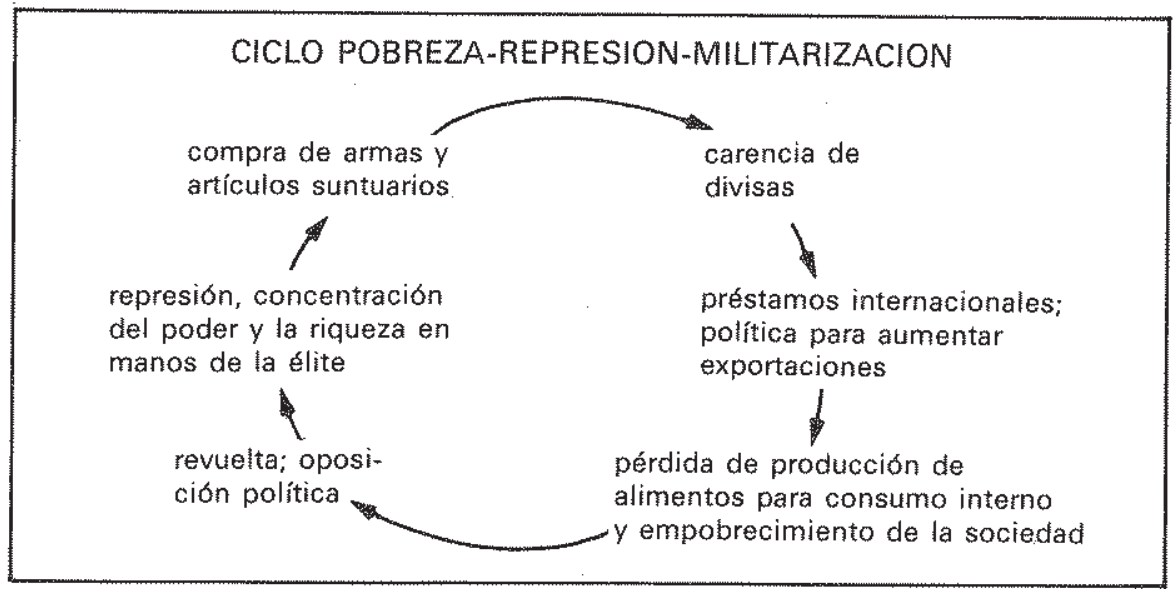

Fuente: Magweta, en Desarrollo, 1982.

\section{Gráfico 2}

\section{LA CARRERA ARMAMENTISTA, EMPOBRECIMIENTO PARA LOS PAISES DESARROLLADOS: EL PARO Y LA INFLACION}

El informe de las Naciones Unidas sobre la relación entre desarme y desarrollo ha dejado bien claro que la humanidad tiene que optar entre continuar adelante con la carrera armamentista o bien avanzar hacia un desarrollo social y económico más equilibrado dentro de un nuevo orden económico y político internacional más viable. Lo que no se puede hacer son las dos cosas (8).

No es cierto que la industria bélica haga aumentar las inversiones, que potencie las ramas civiles de la industria y que haga crecer los puestos de trabajo (9).

Es verdad que se calcula que trabajan, de una manera o de otra, en el aparato militar alrededor de 44.500 .000 personas. Es evidente que el militarismo crea puestos de trabajo. Pero hay que tener en cuenta que:

a) Por culpa de esto, muchos otros sectores de la economía se ven privados de inversiones.

b) Las fábricas de armamento utilizan una tecnología que tiende a ahorrar puestos de trabajo. Según estimaciones del gobierno de los Estados Unidos hechas hace ya algunos años y que cita M. Aguirre, mil millones de dólares destinados a gastos militares crearían 76.000 puestos de trabajo, mientras que dedicados a gastos civiles del gobierno federal crearían 100.000 puestos de trabajo. En conjunto parece que se puede afirmar que el aumento de los gastos públicos militares y la disminución

(8) La relación entre desarme y desarrollo: Naciones Unidas, Nueva York, 1982. Reproducido y editado por el Servicio de Documentación y Difusión de la Asociación para las Naciones Unidas en España. Septiembre, 1984.

(9) Mariano Aguirre, o. c. pág. 161, ss. 
de los gastos públicos no militares harán aumentar el índice de paro.

Los gastos militares son causa del aumento del proceso inflacionario producido por el incremento de la demanda y la inflación de costos. La expansión de la demanda militar coincide con la expansión de la demanda de ciertos sectores civiles, que hará subir todos los precios, pero "el gasto militar no se rige por la efectividad de los costos, sino por la efectividad (bélica) de los productos" (10).

La inflación de costos se manifiesta a través de las tres características siguientes:

a) Los bienes militares se fabrican en las industrias muy avanzadas con índices salariales altos. Con los convenios se pasan a otros sectores industriales con menos productividad $y$ demanda provocando un aumento de inflación.

b) El sector militar - muy monopolítico - tiende a incrementar los costos de manera unilateral.

c) La exportación de armas genera una competencia interestatal. Cada país ha de dedicar más divisas para adquirir armas más caras. La inflación se exporta con los productos bélicos (11).

\section{CAMINOS DE RUPTURA: EL NUEVO MOVIMIENTO PACIFISTA EUROPEO YLOS PROYECTOS ALTERNATIVOS}

\section{El nuevo movimiento pacifista europeo (12)}

El nuevo movimiento pacifista europeo abarca gran variedad de posturas y niveles diversos. Un punto de aglutinamiento fundamental fue la oposición a la instalación de los euromisiles en Europa y la amenaza que esto representaba de convertir al viejo continente en campo de enfrentamiento limitado entre las dos superpotencias.

El movimiento se ha nutrido de contingentes diversos: oposición a la carrera de armamentos, a todo armamento nuclear, a la política de bloques, a la OTAN, antimilitarismo, apoyo a los objetores de conciencia, etc.

Las diferencias son grandes según el nivel. Desde un pacifismo radical hasta un tipo de oposición limitado al armamentismo nuclear.

También los orígenes son diferentes. En Alemania, por ejemplo, se ha ido pasando a los movimientos por la paz desde el ecologismo. En Holanda ha habido un desplazamiento desde los movimientos feministas, no únicamente con el planteamiento de que la paz es un tema urgente que nos afecta a todos, sino también desde el análisis de la institución y el servicio militar como una representación típica de machismo.

(10) Mariano Aguirre, o. c. pág. 168.

(11) Clyde Sanger: Safe sound. Disarmement and development in the 80 's, Zed Pross, Londres, 1982. Cit. a M. Aguirre, o. c. pág. 169.

(12) Cfr. Enrique Gomáriz: Estrategia para la paz. Mayo, núm, 2, noviembre 1982. 
Una división fundamental es la de los partidarios de un desarme unilateral y los defensores de un desarme multilateral.

Los partidarios de un desarme unilateral creen que decisiones como:

a) La no instalación de euromisiles en su país.

b) La desnuclearización total de su país.

c) El desarme unilateral o la desnuclearización de la Europa Occidental. Hay que tomarlas sin esperar que el resto de los países o bloques tomen la misma decisión.

La razones están fundamentadas en la incapacidad de las negocia* ciones internacionales para encontrar un límite al rearme o desarme simultáneo. En la imposibilidad de saber la correlación de fuerzas entre las dos superpotencias, concluyen con la necesidad de romper la dinámica del rearme y dan importancia a la presión que las pequeñas potencias pueden ir ejerciendo sobre las grandes.

Las multilateralistas consideran impolítico el desarme unilateral que provocaría un desequilibrio demasiado brutal. En cambio, su postura, defendida frecuentemente por los expertos en política internacional, creen que es más realista y viable. En esta órbita podríamos incluir incluso "la opción cero" o la eliminación de los Pershing y Cruise en Occidente y de los SS en el Este, situación difícil de aceptar por la Unión Soviética que quedará en manos de los misiles situados en los barcos de superficie o en los submarinos.

La preocupación por un desarme que sólo puede ser multilateral y simultáneo está animada, en muchos autores, por el miedo soviético. El pacifismo europeo de los años treinta, dicen, condujo a no intervenir en la guerra de España y fomentó el rearme desequilibrado de los nazis. También dentro del multilateralismo hay autores que esperan que esta política ayudará a crecer a los movimientos por la paz en el Este. Ya se ve que para llevar adelante un proyecto de desarme hay que encontrar una relación entre las posturas multilateralistas que muchas veces han sido simple "verbalismo" y las posturas unilateralistas, también a veces puramente "testimoniales".

Podríamos enumerar algunos de los aspectos significativos que encontramos en los nuevos movimientos por la paz:

a) La consideración de la guerra nuclear como un fenómeno totalmente nuevo.

b) La configuración en movimientos de masas. Convocan millones de personas en todo ef mundo.

c) Hacen un vínculo cada vez más claro entre la carrera nuclear, el imposible desarrollo de los países del Sur y la crisis del Norte.

d) Se establece una conexión profunda con otros movimiento: ecologistas, feministas, objetores de conciencia. 
e) Muestran una actitud crítica ante las dos superpotencias. Como dice Fred Halliday "los objetivos estratégicos de los movimientos europeos por la paz han de ser dobles: liberar la mitad occidental del continente del sistema militar, que representa el foco más grande de guerra, y a la mitad oriental del sistema público, que representa la negación de las libertades civiles". (13).

Se podría decir que los movimientos por la paz buscan encontrar el "sentido" y la "racionalidad" del mundo actual. Se les acusa de utópicos pero quizá es más utópico pensar que construiremos la paz sentados sobre un polvorín que crece cada minuto y sobre las espaldas de millones de personas que mueren de hambre cada año.

Son bien conocidas las críticas -ideológicas y tendenciosas la mayoría - que se hacen a los movimientos por la paz. Se dice que están financiados por Moscú, que aumentan el peligro de guerra, que ponen en peligro los valores y la defensa de occidente e, incluso, que harán aumentar el paro. Detrás de estas críticas tenemos la hipótesis de una voluntad agresiva de la URSS y la famosa afirmación de "antes muertos que rojos", que únicamente puede tener sentido como una simple y "numantina" opción personal.

\section{Los proyectos alternativos}

La idea fundamental de los proyectos alternativos es romper con la política de bloques, peligro fundamental de una guerra nuclear. Es el intento de mostrar que una política neutralista no es una cosa imposibley que no supone renunciar a la defensa. La defensa puede presentar muchas variantes, desde una defensa militar no nuclear "estrictamente defensiva" hasta la defensa civil y popular no violenta.

Pongamos los ejemplos clásicos: Suiza y Suecia, que no han intervenido en ninguna guerra desde 1815 y que no pertenecen a ningún bloque. Finlandia y Austria, con una neutralidad que empezó después de la Segunda Guerra Mundial. Yugoslavia, que en la época Staliniana rompe con el bloque del Este. El caso extremo de Albania, que rompe con los países del Este, con la China y también con occidente; o la pequeña república de Malta intentando activar una conferencia de seguridad sobre el Mediterráneo. Johan Galtung (14), cuyos análisis seguimos en todo este apartado, se formula una pregunta clave en toda esta cuestión: ¿cuáles son los países más seguros? Su propuesta ordenada de más a menos es la siguiente:

1. Los más seguros: Suiza, Yugoslavia, Albania.

2. Finlandia, Austria, Suecia, Malta.

3. Francia, Grecia, Rumania, Islandia, Irlanda, Chipre, España.

4. Los más inseguros: los integrados en la OTAN o en el Pacto de Varsovia.

(13) Fred Halliday; The Sources of the new Cold War, en Exterminism and Cold War, Verso, 1982. Pág. 322.

(14) Johan Galtung: Hay alternativas. Madrid, Tecnos, 1984. 
Este análisis vendría a decir que el grado de integración dentro de un bloque es directamente proporcional a la inseguridad.

\section{DIMENSIONES QUE PUEDEN DAR SEGURIDAD Y CREDIBILIDAD A LOS PROYECTOS ALTERNATIVOS}

\section{Falta de capacidad militar ofensiva}

Se trata de desplazar las armas convencionales hacia una desaparición de la capacidad ofensiva y el mantenimiento de las armas de gran actividad defensiva.

Es una situación intermedia ante las posturas límite frente a un ataque nuclear: la no resistencia o respuesta con la mutua destrucción asegurada. Evidentemente esta postura no es aceptada por aquellos que están en contra de todo tipo de armamento y contra la violencia en general y propugnan pasar directamente a la defensa no militar y al desarme general. Esto presenta dos grandes dificultades:

a) Una mayoría de la población no cree en la defensa no militar.

b) La historia demuestra que la seguridad no sobrevive automáticamente.

La posición de Galtung sería ir buscando la combinación de esta defensa militar convencional con los otros sistemas de defensa paramilitar, como la guerrilla, y no militar; como la lucha social, el boicot y la desobediencia civil.

\section{No alineación}

Significa un distanciamiento de los bloques militares. Significa que no puede ser utilizado - bases, trabajos nucleares, subordinación - por ninguno de los bloques militares. La tesis del no alineamiento gradual sostiene que éste es un camino para aumentar la seguridad no sólo del país que se desvincula de los bloques sino, poco a poco, del sistema entero.

\section{La fuerza interna del país}

Se trata de la dimensión interna de la seguridad. Esto representaría, según el análisis citado de Galtung, la conquista de una gran autodependencia económica, ecológica, política, militar. Significa una estructuración del país con capacidad suficiente para valerse por sí mismo en tiempo de crisis. Significa intentar la autosuficiencia y que los recursos no se agoten. Desde el punto de vista político quiere decir poder fijar los objetivos propios y la estrategia para conseguirlos.

Desde el punto de vista militar significa capacidad propia para producir armas no ofensivas y posibilidad de organizar la defensa. 
Todos estos elementos tienen que fundamentarse en un gran nivel de cohesión social capaz de una movilización del país.

\section{La utilidad externa}

El país que quiere mantenerse en esta posición ha de demostrar que libre, no alineado, y entero ofrece unos servicios y una disponibilidad más grande que si es dependiente, alineado, conquistado o destruido. La utilidad puede ser política, económica, cultural, humanitaria, ecológica, etc. Se suele citar el caso de Suiza, con una tradición histórica de Conferencias de Paz y Organizaciones Humanitarias hechas senvir por países antagónicos.

\section{CONCLUSION}

Podemos terminar intentando hacer unas breves reflexiones finales:

1. La carrera armamentista, la disuasión y el imposible equilibrio nos pueden llevar a la dèsaparición de las características actuales de nuestro planeta y a la desaparición de todas las formas de vida. Ahora ya se tienen datos suficientes para poder decir esto con una base científica seria.

2. Esta paz aparente basada en la carrera armamentista, y que incluso puede ser un lujo de los países ricos, mata millones de personas al año porfalta de ayuda económica, frena el desarrollo y es una fábrica de parados.

3. No se puede justificar con ningún criterio ético o jurídico, -pensemos en las clásicas teorías de la guerra justa - una guerra actual con las perspectivas y los efectos que hemos citado en este trabajo. La guerra nuclear hay que considerarla un camino cerrado. La indiscriminación y la incontrolabilidad la hacen injustificable éticamente. Jurídicamente hay que considerarla fuera del derecho. $Y$ si no está justificado nunca el uso del armamento nuclear, tampoco pude estar justificada su fabricación y almacenamiento.

4. La política de bloques que fomenta la carrera armamentista y la inestabilidad mundial, contribuye a aumentar el peligro de guerra. No se ve cómo se pueda aceptar la integración y el reforzamiento de una política de bloques que fomentan la fabricación y almacenamiento de unas armas que no se puedan utilizar nunca.

5. Los caminos clásicos del desarme, como son un poder supranacional o los acuerdos diplomáticos, han fracasado. Hay que encontrar caminos alternativos que pueden ir desde una defensa militar convencional hasta una defensa popular no violenta. Los movimientos por la paz que sostienen las posturas más lúcidas y racionales en estos momentos han de ir creando con imaginación estos proyectos alternativos que puedan ser asumidos por una mayoría creciente de la población.

6. Estos proyectos alternativos son difíciles, pero cuando una institución - como la guerra nuclear - se ha vuelto incontrolable hay que 
suprimirla o superarla. Las propuestas de defensa alternativa pueden parecer utópicas a muchos. Probablemente es más utópico pensar que pueda continuar el crecimiento cualitativo y cuantitativo del armamento nuclear y que jamás será utilizado.

7. El Estado Español tiene aún la oportunidad de contribuir a la distensión entre los bloques, es decir, de ponerse al lado de los países y movimientos que buscan una ruptura con la carrera suicida de la humanidad. Esto supone elaborar unas alternativas nuevas de defensa. Pero es evidente que el paso previo es el no alineamiento con ninguno de los dos bloques.

8. Para acabar, no me resisto a hacerlo con el humor de Peridis: "Parece ocurrir con la salida de la OTAN lo del infierno de Dante: Abandonad toda esperanza." Pues bien, incluso dentro de la OTAN, como en otros países de Europa integrados a la Alianza, se ha de continuar la oposición a la política de bloques, a la carrera irracional de armamentos y la búsqueda de unos nuevos modelos de defensa. 